\title{
Constructive role of dissipation for driven coupled bosonic modes
}

\author{
Chaitanya Joshi, ${ }^{1,2, *}$ Mats Jonson, ${ }^{1,3,4}$ Patrik Öhberg, ${ }^{1}$ and Erika Andersson ${ }^{1}$ \\ ${ }^{1}$ SUPA, Institute of Photonics and Quantum Sciences, Heriot-Watt University, Edinburgh EH14 4AS, United Kingdom \\ ${ }^{2}$ SUPA, School of Physics and Astronomy, University of St. Andrews, KY16 9SS, United Kingdom \\ ${ }^{3}$ Department of Physics, University of Gothenburg, SE-412 96 Göteborg, Sweden \\ ${ }^{4}$ Department of Physics, Division of Quantum Phases \& Devices, Konkuk University, Seoul 143-701, Korea
}

(Received 19 April 2013; published 4 June 2013)

\begin{abstract}
We theoretically investigate a system of two coupled bosonic modes subject to both dissipation and external driving. We show that in the steady state the degree of entanglement between the coupled bosonic modes can be enhanced by dissipation. Nonmonotonic dependence of entanglement on the decay rates is observed when the bosonic modes are asymmetrically coupled to their local baths. This counterintuitive result opens a way to better understand the interplay between noise and coherence in continuous-variable systems driven away from equilibrium.
\end{abstract}

DOI: 10.1103/PhysRevA.87.062304

PACS number(s): 03.67.Bg, 03.65.Yz, 03.75.Gg, 42.50.-p

Entanglement is one of the strangest features of quantum mechanics and at the same time a crucial resource for quantum information processing [1-4]. It is well known that superposition states, including entangled states, are extremely sensitive to noise and dissipation. For example, decoherence induced by an environment tends to reduce quantum-coherent superpositions to incoherent mixtures [5], an effect one typically wishes to minimize. Somewhat counterintuitively, however, decoherence can also be used to generate entanglement [6-8]. Schemes to that effect, typically involving atoms coupled to cavity fields, can be modeled as few-level quantum systems coupled to bosonic modes. In this work we investigate the effect of dissipation on a set of coupled bosonic modes, in a setting which does not involve any few-level systems.

Probing quantum aspects in nonequilibrium systems has recently attracted a lot of interest $[6,9,10]$. The inevitable coupling of a quantum system with its environment results in losses, but external pumping can counter the losses. It is thus of interest to study the nonequilibrium physics of dissipative driven quantum systems. Examples include superconducting qubits coupled to microwave resonators, where as well as photon losses microwave photons are added through external pumping [10].

In the current work our aim is to investigate whether dissipation could be used to generate or enhance entanglement between coupled bosonic modes initially prepared in "classical" separable Gaussian states, such as vacuum, coherent, or thermal states. It is, however, not possible to generate entanglement from classical initial states of bosonic modes coupled by only passive, i.e., nonsqueezing, linear interactions. This is because the state of any number of passively coupled bosonic modes that are initially in a classical state will remain classical. This result still applies in the presence of decoherence and noise, if the noise can be modeled via passive coupling to additional bosonic modes. Essentially, what one has, whether in the presence or absence of dissipation, is equivalent to a linear optical network involving

\footnotetext{
*cj30@st-andrews.ac.uk
}

only beam splitters and phase shifters, which cannot generate any entanglement starting from classical states [11].

Here, we are interested in a physical scenario where bosonic modes are coupled via a squeezing interaction and thus the coupling is no longer passive. In this case entanglement between the bosonic modes, initially prepared in classical states, may indeed arise. We propose to counter the effect of losses with an external pump. We shall restrict ourselves to two coupled bosonic modes, subject to both dissipation and external pumping. We have found that dissipation can then increase the degree of entanglement between the modes in the nonequilibrium steady state [12]. The enhancement of steady-state entanglement between coupled bosonic modes is observed when the modes are asymmetrically coupled to their local baths.

We consider a two-mode interaction Hamiltonian

$$
H_{\text {sys }}=\omega_{a} \hat{a}^{\dagger} \hat{a}+\omega_{b} \hat{b}^{\dagger} \hat{b}+\kappa\left(\hat{a}^{\dagger} \hat{b}^{\dagger} e^{-i 2 \omega_{\mathrm{p}} t}+\hat{b} \hat{a} e^{i 2 \omega_{\mathrm{p}} t}\right),
$$

where we have put $\hbar=1$. The above Hamiltonian describes a two-mode nondegenerate parametric down-conversion process, where the modes are commonly known as the signal and idler. The frequency $\omega_{\mathrm{p}}$ is the pump frequency of an arbitrary classical pump field $[13,14]$. When the frequencies $\omega_{a}$ and $\omega_{b}$ add up to twice the classical pump frequency, the result is parametric resonance. Here we consider a more general scenario where $\omega_{a}+\omega_{b} \neq 2 \omega_{\mathrm{p}}$, in which case the above Hamiltonian describes parametric amplification with a detuned pump.

A complete description of any physical system should also take into account the inevitable coupling between the system of interest and the countless degrees of freedom of the external environment. We envisage a physical scenario where each individual bosonic mode is coupled to its local bath, each of which is modeled as a collection of harmonic oscillators and is assumed to be in thermal equilibrium. The Hamiltonian governing the free evolution of each individual bath takes the form

$$
H_{\mathrm{env}}=\sum_{\Omega} \Omega \hat{h}_{1}^{\dagger \Omega} \hat{h}_{1}^{\Omega}+\sum_{\Omega^{\prime}} \Omega^{\prime} \hat{h}_{2}^{\dagger \Omega^{\prime}} \hat{h}_{2}^{\Omega^{\prime}},
$$


where $\hat{h}_{1}^{\dagger \Omega}\left(\hat{h}_{2}^{\dagger \Omega^{\prime}}\right)$ and $\hat{h}_{1}^{\Omega}\left(\hat{h}_{2}^{\Omega^{\prime}}\right)$ create and destroy a boson in mode $\Omega\left(\Omega^{\prime}\right)$ with energy $\Omega\left(\Omega^{\prime}\right)$, respectively.

The interaction Hamiltonian between each individual mode and its respective reservoir is assumed to take the form

$$
\begin{aligned}
H_{\text {int }}= & \sum_{\Omega} \sigma_{\Omega}\left(\hat{h}_{1}^{\dagger \Omega}+\hat{h}_{1}^{\Omega}\right)\left(\hat{a}+\hat{a}^{\dagger}\right) \\
& +\sum_{\Omega^{\prime}} \eta_{\Omega^{\prime}}\left(\hat{h}_{2}^{\dagger \Omega^{\prime}}+\hat{h}_{2}^{\Omega^{\prime}}\right)\left(\hat{b}+\hat{b}^{\dagger}\right) .
\end{aligned}
$$

It should be stressed that in the Hamiltonian $H_{\text {int }}$ above the interaction between the coupled bosonic modes and their respective reservoirs is not simplified using the rotatingwave approximation (RWA) [15]. A detailed analysis of the significance of going beyond the RWA will be presented elsewhere. However, one should note that in a strongly coupled system simplifying the system-environment interaction by using the RWA might lead to spurious results [16].

Thus the density matrix $\rho_{\text {tot }}$ describing the closed-system dynamics of the joint state of the system of interest and the environment is described by a von Neumann equation of the form

$$
\frac{d}{d t} \rho_{\mathrm{tot}}=-i\left[H_{\mathrm{tot}}, \rho_{\mathrm{tot}}\right],
$$

where $H_{\text {tot }}=H_{\text {sys }}+H_{\text {env }}+H_{\text {int }}$. Making a unitary transformation such that $\tilde{H}_{\text {tot }}=\hat{U}_{1}\left(H_{\text {sys }}+\tilde{H}_{\text {env }}+H_{\text {int }}\right) \hat{U}_{1}^{\dagger}$, where $\hat{U}_{1}=e^{i \omega_{\mathrm{p}} t\left(\hat{a}^{\dagger} \hat{a}+\hat{b}^{\dagger} \hat{b}\right)}$, the above von Neumann equation transforms to

$$
\frac{d}{d t} \rho_{\mathrm{tot}}^{1}=-i\left[H_{\mathrm{tot}}^{1}, \rho_{\mathrm{tot}}^{1}\right],
$$

where $\rho_{\text {tot }}^{1}=\hat{U}_{1} \rho_{\text {tot }} \hat{U}_{1}^{\dagger}$ and

$$
\begin{aligned}
H_{\mathrm{tot}}^{1}= & \Delta_{1} \hat{a}^{\dagger} \hat{a}+\Delta_{2} \hat{b}^{\dagger} \hat{b}+\kappa\left(\hat{a}^{\dagger} \hat{b}^{\dagger}+\hat{b} \hat{a}\right) \\
& +\sum_{\Omega} \Omega \hat{h}_{1}^{\dagger \Omega} \hat{h}_{1}^{\Omega}+\sum_{\Omega^{\prime}} \Omega^{\prime} \hat{h}_{2}^{\dagger \Omega^{\prime}} \hat{h}_{2}^{\Omega^{\prime}} \\
& +\sum_{\Omega} \sigma_{\Omega}\left(\hat{h}_{1}^{\dagger \Omega}+\hat{h}_{1}^{\Omega}\right)\left(\hat{a} e^{-i \omega_{\mathrm{p}} t}+\hat{a}^{\dagger} e^{i \omega_{\mathrm{p}} t}\right) \\
& +\sum_{\Omega^{\prime}} \eta_{\Omega^{\prime}}\left(\hat{h}_{2}^{\dagger \Omega^{\prime}}+\hat{h}_{2}^{\Omega^{\prime}}\right)\left(\hat{b} e^{-i \omega_{\mathrm{p}} t}+\hat{b}^{\dagger} e^{i \omega_{\mathrm{p}} t}\right),
\end{aligned}
$$

where $\Delta_{1}=\omega_{a}-\omega_{\mathrm{p}}$ and $\Delta_{2}=\omega_{b}-\omega_{\mathrm{p}}$. The system Hamiltonian then takes the simplified form $H_{\text {sys }}=\Delta_{1} \hat{a}^{\dagger} \hat{a}+$ $\Delta_{2} \hat{b}^{\dagger} \hat{b}+\kappa\left(\hat{a}^{\dagger} \hat{b}^{\dagger}+\hat{b} \hat{a}\right)$, which can now be exactly diagonalized using a nonunitary Bogoliubov transformation, $\hat{a}=$ $\alpha \hat{l}+\beta \hat{m}^{\dagger}$ and $\hat{b}^{\dagger}=\beta \hat{l}+\alpha \hat{m}^{\dagger}$, where $\alpha$ and $\beta$ are complex parameters. To preserve the bosonic commutation relation, we require that $|\alpha|^{2}-|\beta|^{2}=1$, which results in

$$
\begin{gathered}
\alpha=i \sqrt{\frac{1}{2} \sqrt{\frac{\left(\Delta_{1}+\Delta_{2}\right)^{2}}{\left(\Delta_{1}+\Delta_{2}\right)^{2}-4 \kappa^{2}}+\frac{1}{2}}}, \\
\beta=-i \sqrt{\frac{1}{2} \sqrt{\frac{\left(\Delta_{1}+\Delta_{2}\right)^{2}}{\left(\Delta_{1}+\Delta_{2}\right)^{2}-4 \kappa^{2}}}-\frac{1}{2} .}
\end{gathered}
$$

The system Hamiltonian $H_{\text {sys }}$ is then

$$
H_{\text {sys }}=\alpha_{11} \hat{l}^{\dagger} \hat{l}+\alpha_{22} \hat{m}^{\dagger} \hat{m}
$$

where

$$
\begin{aligned}
\alpha_{11}= & \frac{1}{2}\left(\Delta_{1}-\Delta_{2}\right)-\frac{2 \kappa^{2}}{\sqrt{\left(\Delta_{1}+\Delta_{2}\right)^{2}-4 \kappa^{2}}} \\
& +\frac{1}{2}\left(\Delta_{1}+\Delta_{2}\right) \sqrt{\frac{\left(\Delta_{1}+\Delta_{2}\right)^{2}}{\left(\Delta_{1}+\Delta_{2}\right)^{2}-4 \kappa^{2}}}, \\
\alpha_{22}= & -\frac{1}{2}\left(\Delta_{1}-\Delta_{2}\right)-\frac{2 \kappa^{2}}{\sqrt{\left(\Delta_{1}+\Delta_{2}\right)^{2}-4 \kappa^{2}}} \\
& +\frac{1}{2}\left(\Delta_{1}+\Delta_{2}\right) \sqrt{\frac{\left(\Delta_{1}+\Delta_{2}\right)^{2}}{\left(\Delta_{1}+\Delta_{2}\right)^{2}-4 \kappa^{2}}} .
\end{aligned}
$$

In terms of Bogoliubov modes $\hat{l}\left(\hat{l}^{\dagger}\right)$ and $\hat{m}\left(\hat{m}^{\dagger}\right)$, the Hamiltonian (6) can be written as

$$
\begin{aligned}
H_{\mathrm{tot}}^{1}= & \alpha_{11} \hat{l}^{\dagger} \hat{l}+\alpha_{22} \hat{m}^{\dagger} \hat{m}+\sum_{\Omega} \Omega \hat{h}_{1}^{\dagger \Omega} \hat{h}_{1}^{\Omega}+\sum_{\Omega^{\prime}} \Omega^{\prime} \hat{h}_{2}^{\dagger \Omega^{\prime}} \hat{h}_{2}^{\Omega^{\prime}} \\
& +\sum_{\Omega} \sigma_{\Omega}\left(\hat{h}_{1}^{\dagger \Omega}+\hat{h}_{1}^{\Omega}\right)\left[\left(\alpha \hat{l}+\beta \hat{m}^{\dagger}\right) e^{-i \omega_{\mathrm{p}} t}\right. \\
& \left.+\left(\alpha^{*} \hat{l}^{\dagger}+\beta^{*} \hat{m}\right) e^{i \omega_{\mathrm{p}} t}\right]+\sum_{\Omega^{\prime}} \eta_{\Omega^{\prime}}\left(\hat{h}_{2}^{\dagger \Omega^{\prime}}+\hat{h}_{2}^{\Omega^{\prime}}\right) \\
& \times\left[\left(\alpha^{*} \hat{m}+\beta^{*} \hat{l}^{\dagger}\right) e^{-i \omega_{\mathrm{p}} t}+\left(\alpha \hat{m}^{\dagger}+\beta \hat{l}\right) e^{i \omega_{\mathrm{p}} t}\right]
\end{aligned}
$$

In the interaction picture, using the unitary transformation $\hat{U}_{2}=e^{i\left(H_{\mathrm{sys}}+H_{\text {env }}\right) t}, H_{\text {tot }}^{1}$ transforms as $\hat{U}_{2} H_{\text {tot }}^{1} \hat{U}_{2}^{\dagger}$. The systemenvironment joint state then evolves according to

$$
\frac{d}{d t} \tilde{\rho}_{\mathrm{tot}}=-i\left[\tilde{H}_{\mathrm{int}}, \tilde{\rho}_{\mathrm{tot}}\right]
$$

where $\tilde{\rho}_{\text {tot }}=\hat{U}_{2} \rho_{\text {tot }}^{1} \hat{U}_{2}^{\dagger}$ and

$$
\begin{aligned}
\tilde{H}_{\mathrm{int}}= & \sum_{\Omega} \sigma_{\Omega}\left(\tilde{\hat{h}}_{1}^{\dagger \Omega}+\tilde{\hat{h}}_{1}^{\Omega}\right)\left[\left(\alpha \tilde{\hat{l}}+\beta \tilde{\hat{m}}^{\dagger}\right) e^{-i \omega_{\mathrm{p}} t}\right. \\
& \left.+\left(\alpha^{*} \tilde{\hat{l}}^{\dagger}+\beta^{*} \tilde{\hat{m}}\right) e^{i \omega_{\mathrm{p}} t}\right]+\sum_{\Omega^{\prime}} \eta_{\Omega^{\prime}}\left(\tilde{\hat{h}}_{2}^{\dagger \Omega^{\prime}}+\tilde{\hat{h}}_{2}^{\Omega^{\prime}}\right) \\
& \times\left[\left(\alpha^{*} \tilde{\hat{m}}+\beta^{*} \tilde{\hat{l}}^{\dagger}\right) e^{-i \omega_{\mathrm{p}} t}+\left(\alpha \tilde{\hat{m}}^{\dagger}+\beta \tilde{\hat{l}}\right) e^{i \omega_{\mathrm{p}} t}\right],
\end{aligned}
$$

with $\tilde{\hat{x}}=\hat{U} \hat{x} \hat{U}^{\dagger}$. Tracing over the degrees of freedom of the environment, we get a master equation for the reduced density matrix of the two coupled bosonic modes,

$$
\frac{d}{d t} \tilde{\rho}_{\text {sys }}=\operatorname{Tr}_{\text {env }} \frac{d}{d t} \tilde{\rho}_{\text {tot }}=-i \operatorname{Tr}_{\text {env }}\left[\tilde{H}_{\text {int }}, \tilde{\rho}_{\text {tot }}\right] .
$$

For an environment in thermal equilibrium and to first order in the system-environment coupling strength the above equation takes the form

$$
\begin{aligned}
& \frac{d}{d t} \tilde{\rho}_{\mathrm{sys}} \\
& \quad=-\int_{0}^{\infty} \operatorname{Tr}_{\mathrm{env}}\left[\tilde{H}_{\mathrm{int}}(t),\left[\tilde{H}_{\mathrm{int}}\left(t-t^{\prime}\right), \tilde{\rho}_{\mathrm{sys}}(t) \otimes \rho_{\mathrm{env}}(0)\right]\right] d t^{\prime},
\end{aligned}
$$

where to get the final form of the master equation the BornMarkov approximation has been made. 
Expanding the double commutator, explicitly considering terms like $\operatorname{Tr}_{\text {env }}\left[\tilde{H}_{\text {int }}(t) \tilde{\rho}_{\text {sys }}(t) \otimes \rho_{\text {env }}(0) \tilde{H}_{\text {int }}\left(t-t^{\prime}\right)\right]$, and using the secular approximation one gets

$$
\begin{aligned}
\frac{d}{d t} \tilde{\rho}_{\mathrm{sys}}= & i\left\{\left(|\alpha|^{2}\left(\Gamma_{2}+\Gamma_{4}\right)+|\beta|^{2}\left(\gamma_{6}+\gamma_{8}\right)\right) \hat{l}^{\dagger} \hat{l}\right. \\
& \left.+\left[|\alpha|^{2}\left(\gamma_{2}+\gamma_{4}\right)+|\beta|^{2}\left(\Gamma_{6}+\Gamma_{8}\right)\right] \hat{m}^{\dagger} \hat{m}, \tilde{\rho}_{\mathrm{sys}}(t)\right\} \\
& +\left(|\alpha|^{2} \Gamma_{1}+|\beta|^{2} \gamma_{5}\right) \mathcal{L}_{\hat{l}} \tilde{\rho}_{\mathrm{sys}}(t) \\
& +\left(|\alpha|^{2} \Gamma_{3}+|\beta|^{2} \gamma_{7}\right) \mathcal{L}_{\hat{l}^{\dagger}} \tilde{\rho}_{\mathrm{sys}}(t) \\
& +\left(|\alpha|^{2} \gamma_{1}+|\beta|^{2} \Gamma_{5}\right) \mathcal{L}_{\hat{m}} \tilde{\rho}_{\mathrm{sys}}(t) \\
& +\left(|\alpha|^{2} \gamma_{3}+|\beta|^{2} \Gamma_{7}\right) \mathcal{L}_{\hat{m}^{\dagger}} \tilde{\rho}_{\mathrm{sys}}(t),
\end{aligned}
$$

where $\mathcal{L}_{\hat{x}} \rho_{\text {sys }}(t)=2 \hat{x} \rho_{\text {sys }}(t) \hat{x}^{\dagger}-\hat{x}^{\dagger} \hat{x} \rho_{\text {sys }}(t)-\rho_{\text {sys }}(t) \hat{x}^{\dagger} \hat{x}$ and with the set of variables

$$
\begin{aligned}
& \Gamma_{1}+i \Gamma_{2}=\int_{0}^{\infty} d t^{\prime} \sum_{\Omega} \sigma_{\Omega}^{2} e^{i \Omega t^{\prime}} e^{-i\left(\omega_{\mathrm{p}}+\alpha_{11}\right) t^{\prime}}, \\
& \Gamma_{3}+i \Gamma_{4}=\int_{0}^{\infty} d t^{\prime} \sum_{\Omega} \sigma_{\Omega}^{2} e^{i \Omega t^{\prime}} e^{i\left(\omega_{\mathrm{p}}+\alpha_{11}\right) t^{\prime}}, \\
& \Gamma_{5}+i \Gamma_{6}=\int_{0}^{\infty} d t^{\prime} \sum_{\Omega} \sigma_{\Omega}^{2} e^{i \Omega t^{\prime}} e^{i\left(\omega_{\mathrm{p}}-\alpha_{22}\right) t^{\prime}}, \\
& \Gamma_{7}+i \Gamma_{8}=\int_{0}^{\infty} d t^{\prime} \sum_{\Omega} \sigma_{\Omega}^{2} e^{i \Omega t^{\prime}} e^{-i\left(\omega_{\mathrm{p}}-\alpha_{22}\right) t^{\prime}}, \\
& \gamma_{1}+i \gamma_{2}=\int_{0}^{\infty} d t^{\prime} \sum_{\Omega^{\prime}} \eta_{\Omega^{\prime}}^{2} e^{i \Omega^{\prime} t^{\prime}} e^{-i\left(\omega_{\mathrm{p}}+\alpha_{22}\right) t^{\prime}}, \\
& \gamma_{3}+i \gamma_{4}=\int_{0}^{\infty} d t^{\prime} \sum_{\Omega^{\prime}} \eta_{\Omega^{\prime}}^{2} e^{i \Omega^{\prime} t^{\prime}} e^{i\left(\omega_{\mathrm{p}}+\alpha_{22}\right) t^{\prime}}, \\
& \gamma_{5}+i \gamma_{6}=\int_{0}^{\infty} d t^{\prime} \sum_{\Omega^{\prime}} \eta_{\Omega^{\prime}}^{2} e^{i \Omega^{\prime} t^{\prime}} e^{i\left(\omega_{\mathrm{p}}-\alpha_{11}\right) t^{\prime}}, \\
& \gamma_{7}+i \gamma_{8}=\int_{0}^{\infty} d t^{\prime} \sum_{\Omega^{\prime}} \eta_{\Omega^{\prime}}^{2} i e^{i{ }^{\prime} t^{\prime}} e^{-i\left(\omega_{\mathrm{p}}-\alpha_{11}\right) t^{\prime}} .
\end{aligned}
$$

For the sake of simplifying the calculations without compromising the physical insight, we assume that each bath is in thermal equilibrium at zero temperature and with a Drude-Lorentz cutoff for the spectral density, $J_{1}(\Omega) \approx \sum_{x} \sigma_{x}^{2} \delta(x-\Omega) \approx \zeta_{1} \Omega /\left(v_{1}^{2}+\Omega^{2}\right)$ and $J_{2}\left(\Omega^{\prime}\right) \approx$ $\sum_{y} \eta_{y}^{2} \delta\left(y-\Omega^{\prime}\right) \approx \zeta_{2} \Omega^{\prime} /\left(\nu_{2}^{2}+\Omega^{\prime 2}\right)$, respectively, where $\zeta_{1}$ $\left(\zeta_{2}\right)$ is the coupling constant between the first (second) bosonic mode and its local reservoir with $v_{1}$ and $v_{2}$ the cut-off frequencies for the two reservoirs. The above set of integrals can then be analytically solved.

Transforming back from the interaction picture of the free evolution of the normal modes $\hat{l}$ and $\hat{m}$, the master equation takes the form

$$
\begin{aligned}
\frac{d}{d t} \rho_{\mathrm{sys}}= & -i\left[A \hat{l}^{\dagger} \hat{l}+B \hat{m}^{\dagger} \hat{m}, \rho_{\mathrm{sys}}(t)\right]+C \mathcal{L}_{\hat{l}} \rho_{\mathrm{sys}}(t) \\
& +D \mathcal{L}_{\hat{l^{\dagger}}} \rho_{\mathrm{sys}}(t)+E \mathcal{L}_{\hat{m}} \rho_{\mathrm{sys}}(t)+F \mathcal{L}_{\hat{m}^{\dagger}} \rho_{\mathrm{sys}}(t),
\end{aligned}
$$

where

$$
\begin{aligned}
& A=\alpha_{11}-|\alpha|^{2}\left(\Gamma_{2}+\Gamma_{4}\right)-|\beta|^{2}\left(\gamma_{6}+\gamma_{8}\right), \\
& B=\alpha_{22}-|\alpha|^{2}\left(\gamma_{2}+\gamma_{4}\right)-|\beta|^{2}\left(\Gamma_{6}+\Gamma_{8}\right),
\end{aligned}
$$

$$
\begin{aligned}
& C=|\alpha|^{2} \Gamma_{1}, \quad D=|\beta|^{2} \gamma_{7}, \\
& E=|\alpha|^{2} \gamma_{1}, \quad F=|\beta|^{2} \Gamma_{7} .
\end{aligned}
$$

Using standard techniques the above master equation for the density matrix can be converted to a partial differential equation for the quantum characteristic function [15]. Defining a normal-ordered characteristic function $\chi(\epsilon, \eta, t)=\left\langle e^{\epsilon \hat{l}^{\dagger}} e^{-\epsilon^{*} \hat{l}} e^{\eta \hat{m}^{\dagger}} e^{-\eta^{*} \hat{m}}\right\rangle$, the steady state is a thermal state in the normal modes $\hat{l}$ and $\hat{m}$ each with different thermal occupancy, and takes the form $\chi(\epsilon, \eta, t \rightarrow \infty)=$ $e^{-D /(C-D)|\epsilon|^{2}} e^{-F /(E-F)|\eta|^{2}}$. Further defining a normal-ordered characteristic function for the bare modes $\hat{a}$ and $\hat{b}$ as $\chi\left(\epsilon_{a}, \epsilon_{b}\right)=\left\langle e^{\epsilon_{a} \hat{a}^{\dagger}} e^{-\epsilon_{a}^{*} \hat{a}} e^{\epsilon_{b} \hat{b}^{\dagger}} e^{-\epsilon_{b}^{*} \hat{b}}\right\rangle$, and reexpressing the Bogoliubov modes in terms of bare modes $\hat{a}$ and $\hat{b}$, we get for the quantum characteristic function

$$
\begin{aligned}
\chi\left(\epsilon_{a}, \epsilon_{b}, t \rightarrow \infty\right) & \\
= & \exp \left[1 / 2\left(\left|\epsilon_{a}\right|^{2}+\left|\epsilon_{b}\right|^{2}\right)\right] \\
& \times \exp \left[-\left(\frac{D}{C-D}+1 / 2\right)\left|\epsilon_{a} \alpha^{*}-\epsilon_{b}^{*} \beta^{*}\right|^{2}\right] \\
& \times \exp \left[-\left(\frac{F}{E-F}+1 / 2\right)\left|\epsilon_{b} \alpha-\epsilon_{a}^{*} \beta\right|^{2}\right] .
\end{aligned}
$$

This expression for the steady state of two driven coupled bosonic modes is one of the main results of this work. As
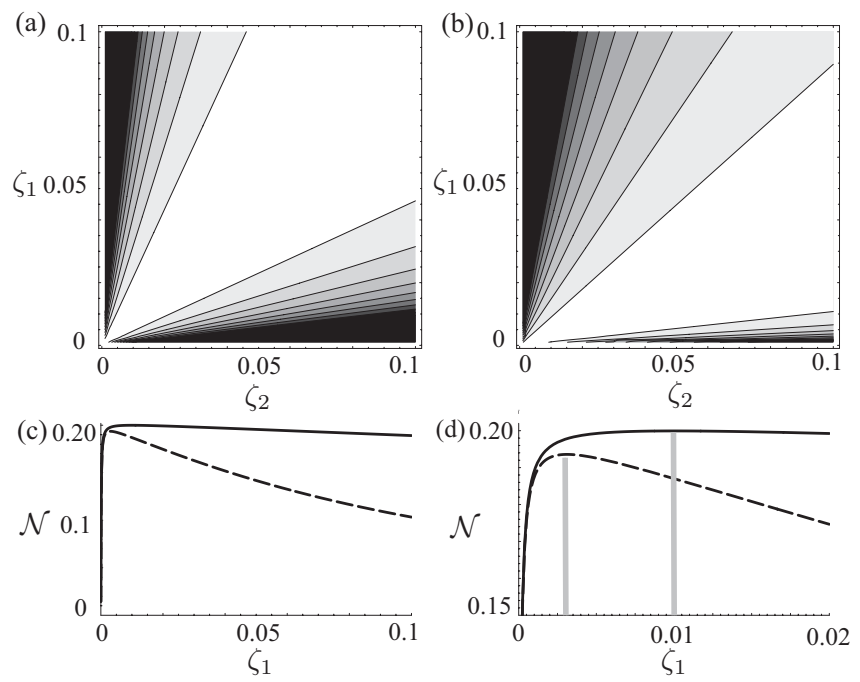

FIG. 1. Steady-state negativity $\mathcal{N}$ calculated as a measure of the quantum entanglement between the studied two coupled bosonic modes and plotted as a function of their decay rates $\zeta_{1}$ and $\zeta_{2}$. In (a) and (b) black indicates regions with minimal and white regions with maximal entanglement. (a) shows the result for two symmetric modes $\omega_{a}=\omega_{b}$, for which maximum entanglement $\mathcal{N}=0.2$ is achieved for $\zeta_{1}=\zeta_{2}$. (b) shows the result for two asymmetric modes $\omega_{b} / \omega_{a}=0.6$, for which the maximum entanglement is obtained for $\zeta_{1} \neq \zeta_{2}$. In (c) $\mathcal{N}$ is plotted as a function of the decay rate $\zeta_{1}$ for the fixed value of $\zeta_{2}=0.01$. One notes that the negativity has a nonmonotonic dependence on $\zeta_{1}$ for both the symmetric (solid line) and the asymmetric (dashed line) cases. In (d), where the same data are plotted on an expanded scale, one sees that the maximum entanglement for the asymmetric case appears for $\zeta_{1}=0.003 \neq \zeta_{2}=0.01$ [marked by thick vertical lines in (d)]. All parameters are given in units of $\omega_{a}$ with $\omega_{\mathrm{p}}=10, v_{1}=v_{2}=1$, and $\kappa=\left|\Delta_{1}+\Delta_{2}\right| / 10=1.84$. 
can be clearly seen, the steady state of the coupled modes depends on the decay strengths of the individual bosonic modes. Interestingly, this dependence on the decay strengths is canceled when the coupling strengths between each individual mode and its respective reservoir are identical.

Now in order to compute the quantum correlations between the coupled bosonic modes $\hat{a}$ and $\hat{b}$, we calculate the logarithmic negativity [3], shown in Fig. 1. We find that it is a nonmonotonic function of their decay rates. Interestingly, we find that when the detuning between the pump and the bosonic modes is chosen such that $\Delta_{1}=\Delta_{2}$, the steady-state entanglement between the modes reaches its maximal value when $\zeta_{1}=\zeta_{2}$, i.e., when the coupling is the same between each mode and its reservoir. This is in stark contrast to the common intuition that environmentally induced decoherence always results in loss of quantum coherence. The results presented in this work can be experimentally tested in an all-optical setup where entangled photon pairs are produced as a result of parametric down-conversion and propagate through two optical fibers with different degrees of loss. It should be noted that in the absence of external pumping, i.e., $\omega_{\mathrm{p}}=0$, it is easy to check that the coefficients $D=F=0$. Thus at zero tem- perature, the steady state of undriven coupled bosonic modes is the ground state, which as expected does not depend on the system-environment coupling strength. Thus it is solely by virtue of external pumping that a nonequilibrium steady state of the form (16) is achieved with nontrivial quantum properties.

In conclusion, we have studied the dissipative dynamics of driven coupled bosonic modes interacting via a two-mode squeezing interaction. The two bosonic modes were coupled to their local baths. At zero temperature the degree of quantum entanglement between coupled bosonic modes is found to be enhanced when the modes are asymmetrically coupled to their local baths. It is tempting to draw a connection between heat flow and the enhancement of quantum correlations in nonequilibrium quantum systems. This merits further investigation.

We gratefully acknowledge helpful discussions with S. M. Barnett, J. Cresser, Michael Hall, and G. J. Milburn. C.J. acknowledges support from the ORS scheme, M.J. partial support from the Swedish VR and the Korean WCU program funded by MEST/NFR (Grant No. R31-2008-000-10057-0), P.Ö. from EPSRC Grant No. EP/J001392/1, and E.A. from EPSRC Grant No. EP/G009821/1.
[1] M. A. Nielsen and I. L. Chuang, Quantum Computation and Quantum Information (Cambridge University Press, Cambridge, 2000).

[2] R. Horodecki, P. Horodecki, M. Horodecki, and K. Horodecki, Rev. Mod. Phys. 81, 865 (1999).

[3] G. Adesso and F. Illuminati, J. Phys. A 40, 7821 (2007).

[4] S. L. Braunstein and H. J. Kimble, Phys. Rev. A 61, 042302 (2000); T. Tyc and B. C. Sanders, ibid. 65, 042310 (2002).

[5] W. H. Zurek, Rev. Mod. Phys. 75, 715 (2003).

[6] M. B. Plenio, S. F. Huelga, A. Beige, and P. L. Knight, Phys. Rev. A 59, 2468 (1999).

[7] A. Beige, S. Bose, D. Braun, S. F. Huelga, P. L. Knight, M. B. Plenio, and V. Vedral, J. Mod. Opt. 47, 2583 (2000).

[8] M. B. Plenio and S. F. Huelga, Phys. Rev. Lett. 88, 197901 (2002).

[9] Michael J. Hartmann, Phys. Rev. Lett. 104, 113601 (2010).
[10] Andrew A. Houck, Hakan E. Türeic, and J. Koch, Nat. Phys. 8, 292 (2012).

[11] M. S. Kim, W. Son, V. Buzek, and P. L. Knight, Phys. Rev. A 65, 032323 (2002).

[12] For the nonequilibrium steady state it holds that $\frac{d}{d t} \rho=\mathcal{L} \rho=0$, where $\rho$ denotes the two-mode density matrix of the coupled bosonic modes written in the rotating frame of the external pump.

[13] G. J. Milburn, A. S. Lane, and D. F. Walls, Phys. Rev. A 27, 2804 (1983).

[14] M. G. A. Paris, in Trends in Quantum Physics, edited by V. Krasnoholovets and F. Columbus (Nova Science Publishers, New York, 2004).

[15] S. M. Barnett and P. M. Radmore, Methods in Theoretical Quantum Optics (Oxford University Press, Oxford, 1997).

[16] J. D. Cresser, J. Mod. Opt. 39, 2187 (1992). 